National Institute of

Standards and Technology

U.S. Department of Commerce

\title{
System and Network Security Acronyms and Abbreviations
}

\author{
Karen Scarfone \\ Victoria Thompson
}


NIST Interagency Report 7581

September 2009
System and Network Security Acronyms and Abbreviations

Karen Scarfone

Victoria Thompson

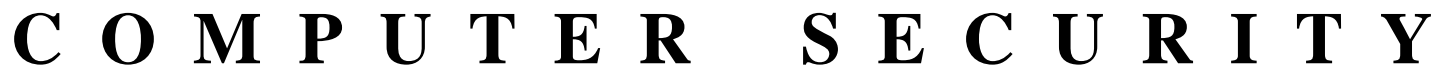

Computer Security Division

Information Technology Laboratory

National Institute of Standards and Technology

Gaithersburg, MD 20899-8930

September 2009

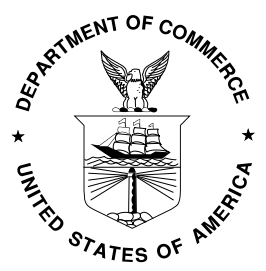

U.S. Department of Commerce

Gary Locke, Secretary

National Institute of Standards and Technology

Patrick D. Gallagher, Deputy Director 


\section{Reports on Computer Systems Technology}

The Information Technology Laboratory (ITL) at the National Institute of Standards and Technology (NIST) promotes the U.S. economy and public welfare by providing technical leadership for the nation's measurement and standards infrastructure. ITL develops tests, test methods, reference data, proof of concept implementations, and technical analysis to advance the development and productive use of information technology. ITL's responsibilities include the development of technical, physical, administrative, and management standards and guidelines for the cost-effective security and privacy of sensitive unclassified information in Federal computer systems. This Interagency Report discusses ITL's research, guidance, and outreach efforts in computer security and its collaborative activities with industry, government, and academic organizations.

\section{National Institute of Standards and Technology Interagency Report 7581 32 pages (Sep. 2009)}

\footnotetext{
Certain commercial entities, equipment, or materials may be identified in this document in order to describe an experimental procedure or concept adequately. Such identification is not intended to imply recommendation or endorsement by the National Institute of Standards and Technology, nor is it intended to imply that the entities, materials, or equipment are necessarily the best available for the purpose.
} 


\section{Acknowledgments}

The authors, Karen Scarfone of the National Institute of Standards and Technology (NIST) and Victoria Thompson of Booz Allen Hamilton, wish to thank their colleagues who reviewed drafts of this report, particularly Liz Lennon and Tim Grance of NIST. Thanks also go to individuals and organizations that submitted suggestions, particularly Tim Kramer, Mark Seecof, Janine Paris, the U.S. Department of Energy, and the U.S. Department of State. The authors also thank their colleagues who created acronym and abbreviation lists for their publications that were subsequently used as sources of information for this report.

\section{Trademark Information}

All names are registered trademarks or trademarks of their respective companies.

\section{Note to Reviewers}

Reviewers are encouraged to submit additional acronyms and abbreviations related to system and network security, particularly for emerging technologies, for consideration as additions to this report. All suggestions and corrections should be sent to securityacronyms@nist.gov. 


\section{Table of Contents}

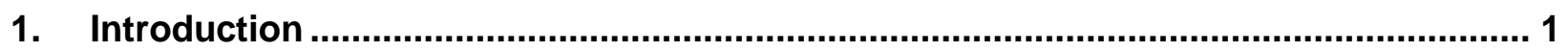

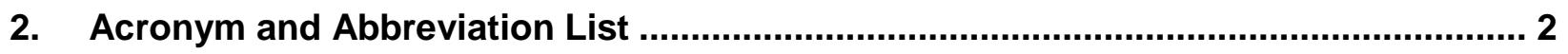

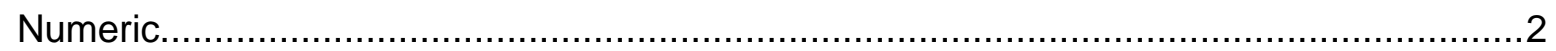

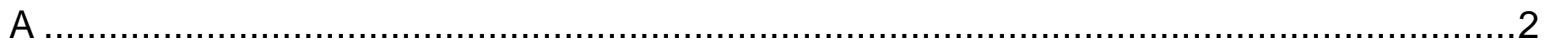

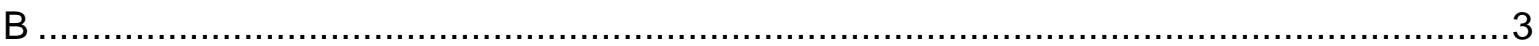

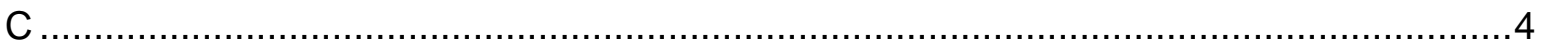

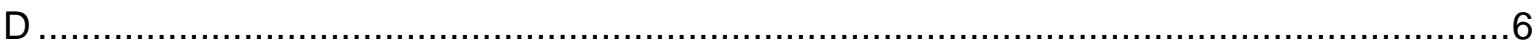

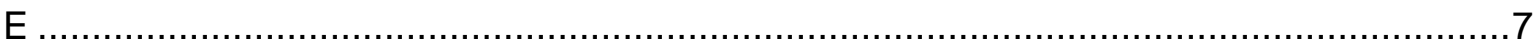

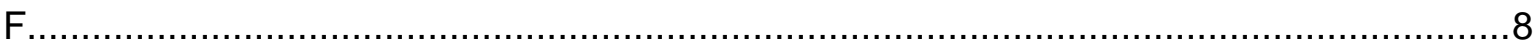

G

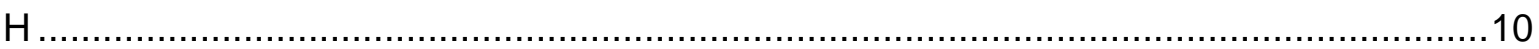

|.

J

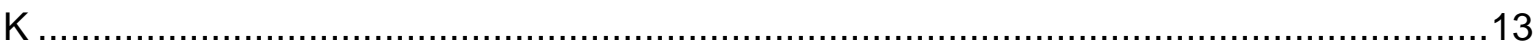

L.

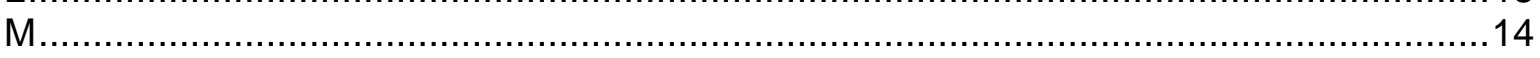

$\mathrm{N}$

O

$\mathrm{P}$.

Q

$\mathrm{R}$

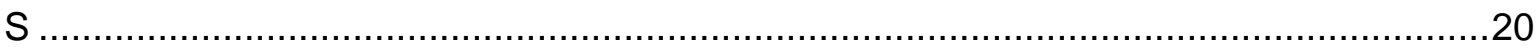

$\mathrm{T}$

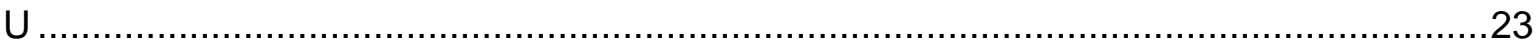

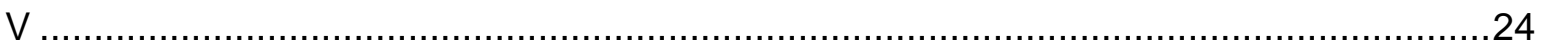

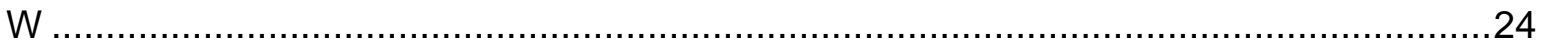

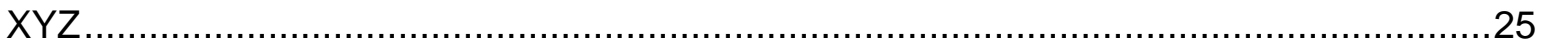

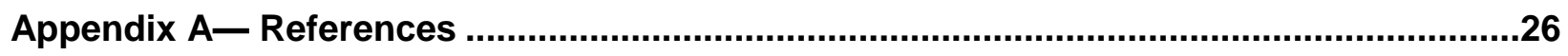

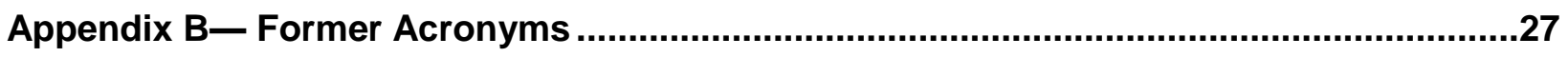




\section{Introduction}

This report contains a list of selected acronyms and abbreviations for system and network security terms with their generally accepted or preferred definitions. It is intended as a resource for federal agencies and other users of system and network security publications.

The capitalization, spelling, and definitions of acronyms and abbreviations frequently vary among publications. It is easy to understand why this happens. While some acronyms and abbreviations (e.g., WWW) have one universally recognized and understood definition within the domain of system and network security, others (e.g., IA, MAC) have multiple valid definitions depending upon the context in which they are used. Some acronyms bear little resemblance to their definitions, such as Modes of Operation Validation System for the Triple DES Algorithm (TMOVS). Others use unexpected capitalization or spelling (e.g., Electronic Business using eXtensible Markup Language [ebXML] and Organisation for Economic Co-operation and Development [OECD]). As a result, acronyms, abbreviations, and their definitions may be inaccurately or inconsistently defined by authors, perpetuating errors and confusing or misleading readers.

This report is meant to help reduce these errors and confusion by providing the generally accepted or preferred definitions of a list of frequently used acronyms and abbreviations. The list does not include all system and network security terms, nor is it a compendium of every acronym and abbreviation found in system and network security documents published by NIST. Readers should refer to each document's list of acronyms and abbreviations (typically found in an appendix) for definitions applicable to that particular document.

The following conventions have been used in the preparation of the list of acronyms and abbreviations in this report.

- Abbreviations and acronyms generally appear in all capital letters, although there are occasional exceptions - for example, meter $(\mathrm{m})$ and decibels referenced to one milliwatt $(\mathrm{dBm})$.

- Technical terms are not capitalized unless they are proper nouns. Names of people, places, and groups, and the titles of protocols, standards, and algorithms are considered proper nouns. For example, certification and accreditation (C\&A) is not capitalized, but Advanced Encryption Standard (AES) is capitalized.

- Collective nouns are not capitalized (e.g., wide area network [WAN]).

- When two or more definitions of the same acronym or abbreviation are given, the acronym or abbreviation is italicized and repeated for each definition. Definitions are listed alphabetically. 


\section{Acronym and Abbreviation List}

This section consists of a list of selected system and network security acronyms and abbreviations, along with their generally accepted definitions. When there are multiple definitions for a single term, the acronym or abbreviation is italicized and each definition is listed separately.

\section{Numeric}

$\begin{array}{ll}\text { 1xRTT } & \text { one times radio transmission technology } \\ \text { 3DES } & \text { Triple Data Encryption Standard } \\ \text { 3G } & \text { 3rd Generation } \\ \text { 3GPP } & \text { 3rd Generation Partnership Project } \\ \text { 3GPP2 } & \text { 3rd Generation Partnership Project 2 }\end{array}$

A

A

AA

AAA

AAAK

AAD

AAR

AAS

ABAC

ACE

ACL

$\mathrm{ACM}$

ACO

AD

$A D$

ADS

AES

AES-CBC

AES-CTR

AFH

A-GPS

AH

AIDC

AIM

AIT

AJAX

AK

AKID

AKM

ALG

ANSI

AP

API address resource record type

$\mathrm{ABAC}$ attribute authority

authentication, authorization, and accounting

authentication, authorization, and accounting key

additional authenticated data

after action report

adaptive antenna system

attribute-based access control

access control entry

access control list

Association for Computing Machinery

authenticated cipher offset

Active Directory

authenticated data

alternate data stream

Advanced Encryption Standard

Advanced Encryption Standard-Cipher Block Chaining

Advanced Encryption Standard-Counter Mode

adaptive frequency hopping

assisted global positioning system

Authentication Header

automatic identification and data capture

Association for Automatic Identification and Mobility

automatic identification technology

Asynchronous JavaScript and XML

authorization key

authorization key identifier

authentication and key management

application layer gateway

American National Standards Institute

access point

application programming interface 


$\begin{array}{ll}\text { APWG } & \text { Anti-Phishing Working Group } \\ \text { ARIN } & \text { American Registry for Internet Numbers } \\ \text { ARP } & \text { Address Resolution Protocol } \\ \text { ARPA } & \text { Advanced Research Projects Agency } \\ \boldsymbol{A S} & \text { authentication server } \\ \boldsymbol{A S} & \text { authentication service } \\ \boldsymbol{A S} & \text { autonomous system } \\ \text { ASC } & \text { Anti-Spyware Coalition } \\ \text { ASC X9 } & \text { Accredited Standards Committee X9 } \\ \text { ASCII } & \text { American Standard Code for Information Interchange } \\ \text { ASLR } & \text { address space layout randomization } \\ \text { ASN } & \text { autonomous system number } \\ \text { ASN.1 } & \text { Abstract Syntax Notation 1 } \\ \text { ASP } & \text { active server pages } \\ \text { ATA } & \text { Advanced Technology Attachment } \\ \text { ATIM } & \text { Announcement Traffic Indication Message } \\ \text { ATM } & \text { asynchronous transfer mode } \\ \text { ATM } & \text { automated teller machine } \\ \text { AV } & \text { antivirus } \\ \text { AVIEN } & \text { Anti-Virus Information Exchange Network } \\ \text { AVP } & \text { attribute-value pair }\end{array}$

B

B2B

$B C P$

$B C P$

BGP

BGP-4

BIA

BIA

BioAPI

BIOS

BITS

BPML

BPSS

BRP

BS

BSC

BSI

BSIA

BSP

BSS

BSSID

BTNS

BTS

BU

BUA business-to-business

best current practice

business continuity plan

Border Gateway Protocol

Border Gateway Protocol 4

Bump-in-the-API

business impact analysis

Biometric Application Programming Interface

basic input/output system

Bump-in-the-Stack

Business Process Modeling Language

business recovery (resumption) plan

base station

base station controller

British Standards Institution

British Security Industry Association

best security practice

basic service set

basic service set identifier

better-than-nothing-security

base transceiver station

binding update

binding update acknowledgement
Business Process Specification Schema 


\begin{tabular}{|c|c|}
\hline C\&A & certification and accreditation \\
\hline$C A$ & certificate authority \\
\hline$C A$ & certification agent \\
\hline$C A$ & certification authority \\
\hline CAC & common access card \\
\hline CAIDA & Cooperative Association for Internet Data Analysis \\
\hline САРТСНА & Completely Automated Public Turing Test to Tell Computers and Humans Apart \\
\hline CARO & Computer Antivirus Research Organization \\
\hline CAVP & Cryptographic Algorithm Validation Program \\
\hline CBC & Cipher Block Chaining \\
\hline CBC-MAC & Cipher Block Chaining Message Authentication Code \\
\hline CBEFF & Common Biometric Exchange File Format \\
\hline $\mathbf{C C}$ & Common Criteria \\
\hline $\mathrm{CCE}^{\mathrm{TM}}$ & Common Configuration Enumeration \\
\hline CCEVS & Common Criteria Evaluation and Validation Scheme \\
\hline CCIPS & Computer Crime and Intellectual Property Section \\
\hline CCK & complementary code keying \\
\hline CCM & Counter Mode with CBC-MAC \\
\hline CCMP & Counter Mode with CBC-MAC Protocol \\
\hline CCRA & Common Criteria Recognition Arrangement \\
\hline CCSS & Common Configuration Scoring System \\
\hline ccTLD & country code top-level domain \\
\hline$C D$ & checking disabled \\
\hline$C D$ & compact disc \\
\hline CDFS & compact disc file system \\
\hline CDMA & code division multiple access \\
\hline CD-R & compact disc-recordable \\
\hline CD-ROM & compact disc-read only memory \\
\hline CD-RW & compact disc-rewritable \\
\hline CEO & chief executive officer \\
\hline CERIAS & Center for Education and Research in Information Assurance and Security \\
\hline CERT & computer emergency response team \\
\hline CERT®/CC & CERT® Coordination Center \\
\hline $\mathbf{C F}$ & CompactFlash® \\
\hline CFAA & Computer Fraud and Abuse Act \\
\hline CFB & Cipher Feedback \\
\hline CFI & computer and financial investigations \\
\hline CFR & Code of Federal Regulations \\
\hline CFTT & computer forensics tool testing \\
\hline CGA & cryptographically generated addresses \\
\hline CGI & Common Gateway Interface \\
\hline CHAP & Challenge-Handshake Authentication Protocol \\
\hline CHUID & cardholder unique identifier \\
\hline CIDR & Classless Inter-Domain Routing \\
\hline CIFS & Common Internet File System \\
\hline CIO & chief information officer \\
\hline CIP & critical infrastructure protection \\
\hline CIPC & Critical Infrastructure Protection Committee \\
\hline
\end{tabular}




\author{
CIPSEA \\ CIRC \\ CIRC \\ CIRDB \\ CIRT \\ CIS \\ CISO \\ CLF \\ CLI \\ CLR \\ cm \\ CMA \\ CMAC \\ CME \\ CMOS \\ CMS \\ CMS \\ CMSS \\ CMVP \\ CN \\ CN \\ CNSS \\ CNSSI \\ CoA \\ codec \\ COI \\ COM \\ COOP \\ COPPA \\ CORBA® \\ COTS \\ CP \\ CP \\ CPE $^{\mathrm{TM}}$ \\ CPI \\ CPNI \\ CPS \\ CPU \\ CRAM \\ CRC \\ CRL \\ CS \\ CSIA \\ CSIRC \\ CSIRT \\ CSO \\ CSO \\ CSP \\ CSR \\ CSRC \\ CSRDA \\ Confidential Information Protection and Statistical Efficiency Act \\ computer incident response capability \\ computer incident response center \\ CERIAS Incident Response Database \\ computer incident response team \\ Center for Internet Security \\ chief information security officer \\ common log format \\ command line interface \\ common language runtime \\ centimeter \\ Certificate Management Authority \\ Cipher-based Method Authentication Code \\ Common Malware Enumeration \\ complementary metal oxide semiconductor \\ Centers for Medicare and Medicaid Services \\ Cryptographic Message Syntax \\ Common Misuse Scoring System \\ Cryptographic Module Validation Program \\ common name \\ correspondent node \\ Committee on National Security Systems \\ Committee on National Security Systems Instruction \\ care-of address \\ coder/decoder \\ conflict of interest \\ Component Object Model \\ continuity of operations \\ Children's Online Privacy Protection Act \\ Common Object Request Broker Architecture \\ commercial off-the-shelf \\ certificate policy \\ contingency plan \\ Common Platform Enumeration \\ compression parameter index \\ Centre for the Protection of National Infrastructure \\ certification practice statement \\ central processing unit \\ challenge-response authentication mechanism \\ cyclic redundancy check \\ certificate revocation list \\ client/server \\ Cyber Security Industries Alliance \\ computer security incident response capability \\ computer security incident response team \\ chief security officer \\ computer security object \\ Credentials Service Provider \\ certificate signing request \\ Computer Security Resource Center \\ Cyber Security Research and Development Act of 2002
}


CSS

CSV

CTO

CTR

CVE

CVSS

CWE

cascading style sheet

comma-separated values

chief technology officer

counter mode encryption

Common Vulnerabilities and Exposures

Common Vulnerability Scoring System

Common Weakness Enumeration

DA

$D A A$

$D A A$

DAC

DAD

DAML

D-AMPS

DAO

DARPA

dBm

DBMS

DC

DCE

DCOM

DCS

DDMS

DDoS

DEA

DEP

DES

DFS

DFS

DH

DHAAD

DHCP

DHCPv6

DHS

DIMS

DISA

DLL

DMA

DMZ

DN

$\underline{D N}$

DNP

DNS

DNSBL

DNSSEC

DOC

DoD

destination address

designated accrediting authority

designated approving authority

discretionary access control

duplicate address detection

DARPA Agent Markup Language

Digital Advanced Mobile Phone Service

Data Access Object

Defense Advanced Research Projects Agency

decibels referenced to one milliwatt

database management system

domain controller

Distributed Computing Environment

Distributed Component Object Model

distributed control system

Department of Defense Metadata Specification

distributed denial of service

Data Encryption Algorithm

Data Execution Prevention

Data Encryption Standard

Distributed File System

dynamic frequency selection

Diffie-Hellman

Dynamic Home Agent Address Discovery

Dynamic Host Configuration Protocol

Dynamic Host Configuration Protocol for Internet Protocol v6

U.S. Department of Homeland Security

Digital Identity Management Service

U.S. Defense Information Systems Agency

dynamic link library

direct memory access

demilitarized zone

distinguished name

domain name

Distributed Network Protocol

domain name system

Domain Name System Blacklist

Domain Name System Security Extensions

U.S. Department of Commerce

U.S. Department of Defense 


DOE
DOI
DOJ
DOM
DoS
DPA
DRA
DRM
DRP
DS
DS
DS Field
DSA
DSL
DSML
DSN
DSOD
DSS
DSTM
DTC
DTD
DTR
DUID
DVD
DVD-R
DVD-ROM
DVD-RW

\section{E}

EAL

EAP

EAP-FAST

EAPOL

EAPOL-KCK

EAPOL-KEK

EAP-TLS

EAP-TTLS

EBGP

ebXML

EC2N

ECB

ECC

ECDH

ECDSA

ECM

ECP

ECPA

EDGE

EDI
U.S. Department of Energy

domain of interpretation

U.S. Department of Justice

Document Object Model

denial of service

differential power analysis

data recovery agent

digital rights management

disaster recovery plan

Delegation Signer

distribution system

differentiated services field

Digital Signature Algorithm

digital subscriber line

Directory Services Markup Language

delivery status notification

dynamic separation of duty

Digital Signature Standard

Dual Stack Transition Mechanism

Distributed Transaction Coordinator

Document Type Definition

derived test requirement

DHCP unique identifier

digital video disc

digital video disc - recordable

digital video disc - read only memory

digital video disc - rewritable

evaluation assurance level

Extensible Authentication Protocol

Extensible Authentication Protocol-Flexible Authentication via Secure Tunneling

Extensible Authentication Protocol Over LAN

Extensible Authentication Protocol Over LAN Key Confirmation Key

Extensible Authentication Protocol Over LAN Key Encryption Key

Extensible Authentication Protocol-Transport Layer Security

Extensible Authentication Protocol-Tunneled Transport Layer Security

Exterior Border Gateway Protocol

Electronic Business using eXtensible Markup Language

Elliptic Curve over G[2N]

Electronic Codebook (mode)

Elliptic Curve Cryptography

Elliptic Curve Diffie-Hellman

Elliptic Curve Digital Signature Algorithm

Enterprise Configuration Manager

Encryption Control Protocol

Electronic Communications Privacy Act

Enhanced Data rates for GSM Evolution

electronic data interchange 


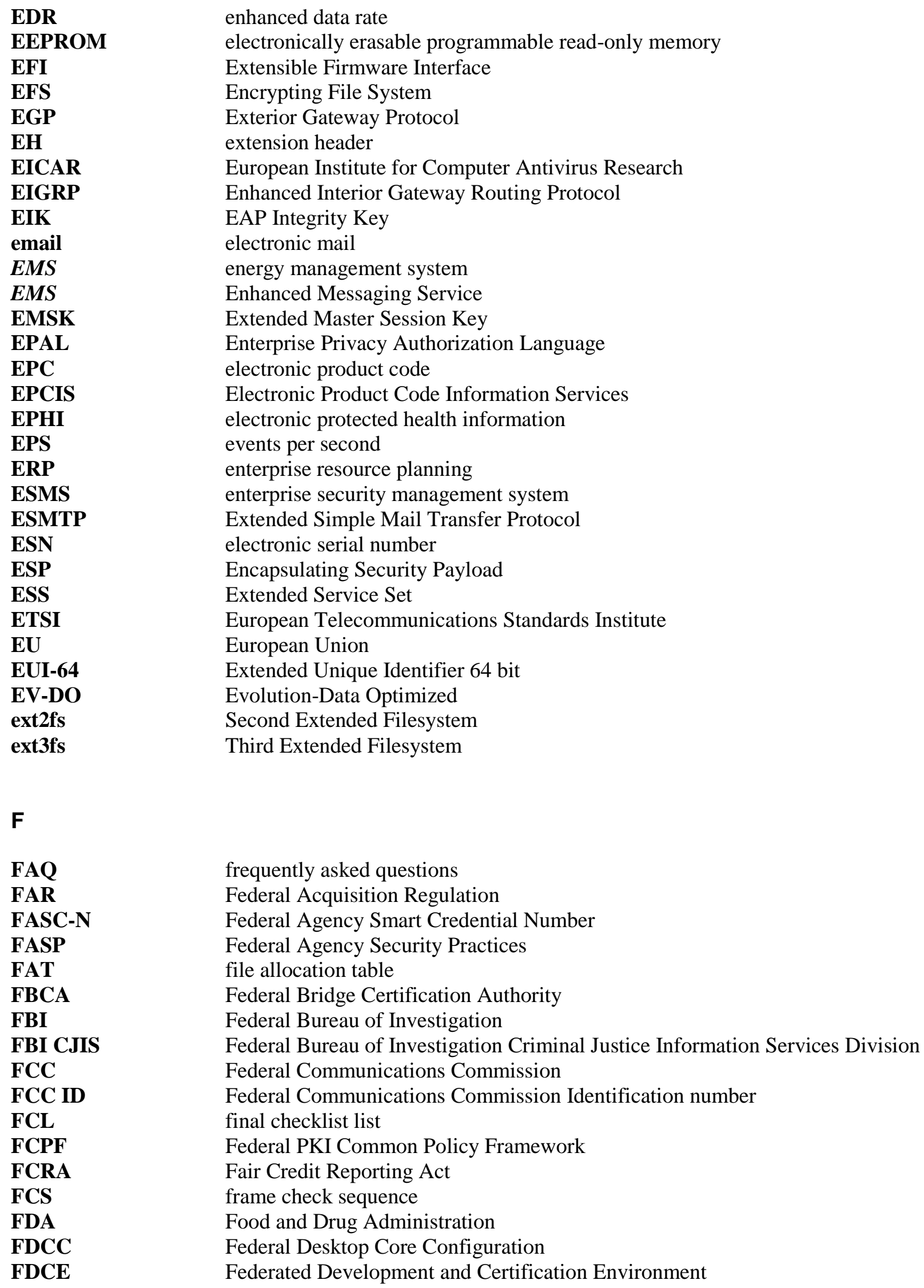

enhanced data rate

electronically erasable programmable read-only memory

Extensible Firmware Interface

Encrypting File System

Exterior Gateway Protocol

extension header

European Institute for Computer Antivirus Research

Enhanced Interior Gateway Routing Protocol

EAP Integrity Key

electronic mail

energy management system

Enhanced Messaging Service

Extended Master Session Key

Enterprise Privacy Authorization Language

electronic product code

Electronic Product Code Information Services

electronic protected health information

events per second

enterprise resource planning

enterprise security management system

Extended Simple Mail Transfer Protocol

electronic serial number

Encapsulating Security Payload

Extended Service Set

European Telecommunications Standards Institute

European Union

Extended Unique Identifier 64 bit

Evolution-Data Optimized

Second Extended Filesystem

Third Extended Filesystem

frequently asked questions

Federal Acquisition Regulation

Federal Agency Smart Credential Number

Federal Agency Security Practices

file allocation table

Federal Bridge Certification Authority

Federal Bureau of Investigation

Federal Bureau of Investigation Criminal Justice Information Services Division

Federal Communications Commission

Federal Communications Commission Identification number

final checklist list

Federal PKI Common Policy Framework

Fair Credit Reporting Act

frame check sequence

Food and Drug Administration

Federal Desktop Core Configuration

Federated Development and Certification Environment 


\begin{tabular}{|c|c|}
\hline FDE & full disk encryption \\
\hline FDIC & Federal Deposit Insurance Corporation \\
\hline FEA & Federal Enterprise Architecture \\
\hline FEK & file encryption key \\
\hline FFMIA & Federal Financial Management Improvement Act \\
\hline FHSS & frequency hopping spread spectrum \\
\hline FIB & forwarding information base \\
\hline FICC & Federal Identity Credentialing Committee \\
\hline FIPS & Federal Information Processing Standards \\
\hline FIRST TM $^{\mathrm{TM}}$ & Forum of Incident Response and Security Teams \\
\hline FISCAM & Federal Information System Controls Audit Manual \\
\hline FISMA & Federal Information Security Management Act of 2002 \\
\hline FISSEA & Federal Information Systems Security Educators' Association \\
\hline FLETC & Federal Law Enforcement Training Center \\
\hline FMR & false match rate \\
\hline FMS & Fluhrer-Mantin-Shamir \\
\hline FNMR & false non match rate \\
\hline FOIA & Freedom of Information Act \\
\hline FPC & Federal Preparedness Circular \\
\hline FPKI & Federal Public Key Infrastructure \\
\hline FPKIA & Federal Public Key Infrastructure Architecture \\
\hline FPKIPA & Federal Public Key Infrastructure Policy Authority \\
\hline FQDN & fully qualified domain name \\
\hline FRR & false rejection rate \\
\hline FSO & field security office \\
\hline FTC & Federal Trade Commission \\
\hline FTCA & Federal Trade Commission Act \\
\hline FTP & File Transfer Protocol \\
\hline FUS & Fast User Switching \\
\hline $\mathbf{F Y}$ & fiscal year \\
\hline \multicolumn{2}{|l|}{ G } \\
\hline GAO & U.S. Government Accountability Office \\
\hline GB & gigabyte \\
\hline GFAC & generalized framework for access control \\
\hline GFIRST & Government Forum of Incident Response and Security Teams \\
\hline GHz & gigahertz \\
\hline GIG & Global Information Grid \\
\hline GINA & graphical identification and authentication \\
\hline GKEK & Group Key Encryption Key \\
\hline GLB or GLBA & Gramm-Leach-Bliley Act \\
\hline GMK & Group Master Key \\
\hline GnuPG & GNU Privacy Guard \\
\hline GOTS & government off-the-shelf \\
\hline GPL & general public license \\
\hline GPMC & Group Policy Management Console \\
\hline GPO & Group Policy Object \\
\hline GPRS & general packet radio service \\
\hline GPS & global positioning system \\
\hline
\end{tabular}


GR

GRE

GRS

GS1

GSA

GSM

GTC

GTEK

GTK

gTLD

GTSM

GUI

H

HA

HA

HAG

HCI

HERF

HERO

HERP

HF

HFS

HHS

HINFO

HIP

HIPAA

HIPERLAN

HKLM

HL7

HMAC

HMI

HPA

HPFS

HR

HSARPA

HSPD

HTCIA

HTCP

HTML

HTTP

HTTPS

Hz graceful restart

Generic Routing Encapsulation

General Records Schedule

Global Standards One

U.S. General Services Administration

Global System for Mobile Communications

Generic Token Card

group traffic encryption key

group temporal key

generic top-level domain

Generalized TTL Security Mechanism

graphical user interface

high availability

home agent

high assurance guard

host controller interface

hazards of electromagnetic radiation to fuel

hazards of electromagnetic radiation to ordnance

hazards of electromagnetic radiation to personnel

high frequency

Hierarchical File System

U.S. Department of Health and Human Services

host information

Host Identity Protocol

Health Insurance Portability and Accountability Act

high-performance radio local area network

HKEY_Local_Machine

Health Level Seven

keyed-hash message authentication code

human-machine interface

host protected area

High-Performance File System

human resources

Homeland Security Advanced Research Projects Agency

Homeland Security Presidential Directive

High Technology Crime Investigation Association

Hyper Text Caching Protocol

Hypertext Markup Language

Hypertext Transfer Protocol

Secure Hypertext Transfer Protocol

hertz 
I\&A

I/O

I3P

IA

IAB

IACIS ${ }^{\circledR}$

IAIP

IANA

IAO

IATF

IBC

IBE

iBGP

IBMJSSE

IBSS

IC3

ICAMP

ICANN

ICCID

ICCP

ICF

ICMP

ICP

ICS

ICS

ICSA

ICV

ID

IDART ${ }^{T M}$

IDE

IDE

IDEA

iDEN

ID-FF

IDMEF

IDMS

IDPS

IDS

ID-SIS

ID-WSF

ID-WSF DST

IE

IEC

IED

IEEE-SA

IESG

IETF

IETF BCP

IETF RFC identification and authentication

input/output

Institute for Information Infrastructure Protection

information assurance

Internet Architecture Board

International Association of Computer Investigative Specialists

Information Analysis and Infrastructure Protection

Internet Assigned Numbers Authority

information assurance officer

Information Assurance Technical Framework

iterated block cipher

identity-based encryption

Internal Border Gateway Protocol

IBM Java Secure Socket Extension

independent basic service set

Internet Crime Complaint Center

Incident Cost Analysis and Modeling Project

Internet Corporation for Assigned Names and Numbers

Integrated Circuit Card Identification

Inter-control Center Communications Protocol

Internet Connection Firewall

Internet Control Message Protocol

Internet Cache Protocol

industrial control system

Internet Connection Sharing

International Computer Security Association

integrity check value

identification

Information Design Assurance Red Team

integrated development environment

Integrated Drive Electronics

International Data Encryption Algorithm

Integrated Digital Enhanced Network

Identity Federation Framework

Intrusion Detection Message Exchange Format

identity management system

intrusion detection and prevention system

intrusion detection system

Identity Service Interface Specifications

Identity Web Services Framework

Identity Web Services Framework Data Services Template

Internet Explorer

International Electrotechnical Commission

intelligent electronic device

IEEE Standards Association

Internet Security Steering Group

Internet Engineering Task Force

Internet Engineering Task Force Best Current Practice

Internet Engineering Task Force Request for Comments 


\begin{tabular}{|c|c|}
\hline IGMP & Internet Group Management Protocol \\
\hline IGP & interior gateway protocol \\
\hline IID & interface identifier \\
\hline IIF & information in identifiable form \\
\hline IIHI & individually identifiable health information \\
\hline IIS & Internet Information Services \\
\hline IKE & Internet Key Exchange \\
\hline IM & instant messaging \\
\hline IMAP & Internet Message Access Protocol \\
\hline IMEI & International Mobile Equipment Identity \\
\hline IMSI & International Mobile Subscriber Identity \\
\hline INCITS & InterNational Committee for Information Technology Standards \\
\hline IP & Internet Protocol \\
\hline IPA & initial privacy assessment \\
\hline IPComp & Internet Protocol Payload Compression Protocol \\
\hline IPng & Internet Protocol Next Generation \\
\hline IPS & intrusion prevention system \\
\hline IPsec & Internet Protocol Security \\
\hline IPv4 & Internet Protocol version 4 \\
\hline IPv6 & Internet Protocol version 6 \\
\hline IPX & Internet Packet Exchange \\
\hline IR & infrared \\
\hline IR & interagency report \\
\hline IRC & Internet Relay Chat \\
\hline IrDA® & Infrared Data Association ${ }^{\circledR}$ \\
\hline IRQ & interrupt request line \\
\hline IRS & Internal Revenue Service \\
\hline IRTF & Internet Research Task Force \\
\hline IS & information system \\
\hline ISA & interconnection security agreement \\
\hline ISA & International Society of Automation \\
\hline ISAC & information sharing and analysis center \\
\hline ISAKMP & Internet Security Association and Key Management Protocol \\
\hline ISAP & Information Security Automation Program \\
\hline ISAPI & Internet Server Application Programming Interface \\
\hline ISATAP & Intra-Site Automatic Tunnel Addressing Protocol \\
\hline ISF & Information Security Forum \\
\hline ISID & Industrial Security Incident Database \\
\hline IS-IS & Intermediate System-to-Intermediate System \\
\hline ISM & industrial, scientific, and medical \\
\hline ISM & information security marking \\
\hline ISMS & information security management system \\
\hline ISO & International Organization for Standardization \\
\hline ISP & Internet service provider \\
\hline ISSEA & International Systems Security Engineering Association \\
\hline ISSO & information systems security officer \\
\hline ISSPM & information systems security program manager \\
\hline IT & information technology \\
\hline ITAA & Information Technology Association of America \\
\hline ITF & Interrogator Talks First \\
\hline ITL & Information Technology Laboratory \\
\hline
\end{tabular}


ITU

ITU-T

IUT

IV

$\mathbf{J}$

Java EE

JAXR

JFFS2

JIT

JPEG

JRE

JSM

JSP

JSSE

JTAG

JTC1

JVM

K

KB

Kbps

KDC

KEK

KG

KGD

kHz

KINK

KSG

KSK

L

L2CAP

L2F

L2TP

L2VPN

L3VPN

LACNIC

LAN

LCD

LDA

LDAP

LED
International Telecommunications Union

International Telecommunications Union-Telecommunication Standardization

Sector

implementation under test

initialization vector

Java Platform, Enterprise Edition

Java API for XML Registries

Journaling Flash File System, version 2

just-in-time

Joint Photographic Experts Group

Java Runtime Environment

Java Security Manager

Java Server Pages

Java Secure Socket Extension

Joint Test Action Group

Joint Technical Committee 1 (International Organization for Standardization

[ISO]/International Electrotechnical Commission [IEC])

Java Virtual Machine

kilobyte

kilobit per second

key distribution center

key encryption key

key generator

key generation and distribution

kilohertz

Kerberized Internet Negotiation of Keys

key stream generator

key signing key

Logical Link Control and Adaptation Protocol

Layer 2 Forwarding

Layer 2 Tunneling Protocol

Layer 2 Virtual Private Network

Layer 3 Virtual Private Network

Latin American and Caribbean IP Addresses Registry

local area network

liquid crystal display

local delivery agent

Lightweight Directory Access Protocol

light emitting diode 


$\begin{array}{ll}\text { LF } & \text { low frequency } \\ \text { LFSR } & \text { linear feedback shift register } \\ \text { LIR } & \text { local Internet registry } \\ \text { LM } & \text { LAN Manager } \\ \text { LMP } & \text { Link Manager Protocol } \\ \text { LOC } & \text { location (DNS record) } \\ \text { LOS } & \text { line-of-sight } \\ \text { LRA } & \text { Local Registration Authority } \\ \text { LUA } & \text { limited user account }\end{array}$

\section{M}

$\mathbf{m}$

meter

$M A C$

mandatory access control

media access control (layer)

$M A C$

Medium Access Control

$M A C$

message authentication code

MAF

multi-mode authentication framework

MAN

MAPS

metropolitan area network

MB

Mail Abuse Prevention System

megabyte

Mbps

megabits per second

MBR

MBSA

master boot record

MD

ME

MED

MEP

MES

MHz

MIB

MIC

MIC

MIC

MIKEY

MIME

MIMO

MIN

Mini SD

MIP

MitM

MLD

$M M C$

$M M C$

MMCmobile

MMS

MN

MO

MOA

MOBIKE

Microsoft Baseline Security Analyzer

message digest

mobile equipment

multi-exit discriminator

message exchange pattern

manufacturing execution system

megahertz

management information base

mandatory integrity control

message integrity check

message integrity code

Multimedia Internet KEYing

Multipurpose Internet Mail Extensions

multiple-input, multiple-output

mobile identification number

mini secure digital

Mobile Internet Protocol

man-in-the-middle (attack)

Multicast Listener Discovery

Microsoft Management Console

MultiMediaCard

MultiMediaCard Mobile

Multimedia Messaging Service

mobile node

magneto-optical

memorandum of agreement

IKEv2 Mobility and Multihoming Protocol 


\begin{tabular}{ll} 
MODP & modular exponential \\
MOSS & MIME Object Security Services \\
MOU & memorandum of understanding \\
MOVS & Modes of Operation Validation System \\
MPA & Mobile Prefix Advertisement \\
MPLS & multiprotocol label switching \\
MPS & Mobile Prefix Solicitation \\
MQV & Menezes-Qu-Vanstone \\
MRI & magnetic resonance imaging \\
MS & Microsoft \\
MS & mobile subscriber \\
MSC & mobile switching center \\
MS-CHAP & Microsoft Challenge Handshake Authentication Protocol \\
MS-DOS & Microsoft Disk Operating System \\
MSDP & Multicast Source Discovery Protocol \\
MSEC & multicast security \\
MSEL & Master Scenario Events List \\
MSIL & Microsoft Intermediate Language \\
MSISDN & Mobile Subscriber Integrated Services Digital Network \\
MSK & master session key \\
MSKB & Microsoft Knowledge Base \\
MSSP & managed security services provider \\
MSWG & Metadata Standards Working Group \\
MTA & mail transfer agent \\
MTM & Mobile Trusted Module \\
MTU & master telemetry unit \\
MTU & master terminal unit \\
MTU & maximum transmission unit \\
MUA & mail user agent \\
mW & milliwatt \\
MX & mail exchanger \\
\hline
\end{tabular}

$\mathbf{N}$

NA

NAC

NACI

NAP

NARA

NAS

NAT

NAT-PT

NAT-T

NBA

NBAD

NCES

NCP

NCSD

NCSI

ND

Neighbor Advertisement

network access control

National Agency Check and Inquiries

Network Access Protection

National Archives and Records Administration

network access server

network address translation

network address translation-protocol translation

network address translation traversal

network behavior analysis

network behavior anomaly detection

NetCentric Enterprise Services

National Checklist Program

National Cyber Security Division

NIST National Center for Standards and Certification Information

Neighbor Discovery 


\begin{tabular}{|c|c|}
\hline NDAC & nondiscretionary access control \\
\hline NetBEUI & NetBIOS Extended User Interface \\
\hline NetBIOS & Network Basic Input/Output System \\
\hline NetBT & NetBIOS over TCP/IP \\
\hline NFAT & network forensic analysis tool \\
\hline NFC & near field communication \\
\hline NFS & network file system \\
\hline NFS & Network File Sharing \\
\hline NH & next header \\
\hline NIAC & National Infrastructure Advisory Council \\
\hline NIAP & National Information Assurance Partnership \\
\hline NIC & network interface card \\
\hline NICC & National Infrastructure Coordinating Center \\
\hline NIJ & National Institute of Justice \\
\hline NIPC & National Infrastructure Protection Center \\
\hline NIS & Network Information System \\
\hline NISAC & National Infrastructure Simulation and Analysis Center \\
\hline NISCC & National Infrastructure Security Co-ordination Centre \\
\hline NIST & National Institute of Standards and Technology \\
\hline NISTIR & National Institute of Standards and Technology Interagency Report \\
\hline NLOS & non-line-of-sight \\
\hline NPIVP & NIST Personal Identity Verification Program \\
\hline NPPI & nonpublic personal information \\
\hline$N S$ & name server \\
\hline$N S$ & Neighbor Solicitation \\
\hline NSA & National Security Agency \\
\hline NSAPI & Netscape Server Application Programming Interface \\
\hline NSEC & Next Secure \\
\hline NSI & national security information \\
\hline NSRL & National Software Reference Library \\
\hline NSS & Network Security Services \\
\hline NSTB & National SCADA Test Bed \\
\hline NSTISSC & $\begin{array}{l}\text { National Security Telecommunications and Information Systems Security } \\
\text { Committee }\end{array}$ \\
\hline NSTISSI & $\begin{array}{l}\text { National Security Telecommunications and Information Systems Security } \\
\text { Instruction }\end{array}$ \\
\hline NTFS & New Technology File System \\
\hline NTLM & NT LAN Manager \\
\hline NTP & Network Time Protocol \\
\hline NTTAA & National Technology Transfer and Advancement Act of 1995 \\
\hline NUD & Neighbor Unreachability Detection \\
\hline NVD & National Vulnerability Database \\
\hline NVLAP & National Voluntary Laboratory Accreditation Program \\
\hline NW3C & National White Collar Crime Center \\
\hline $\mathbf{N X}$ & no execute \\
\hline
\end{tabular}




\begin{tabular}{|c|c|}
\hline & \\
\hline OCC & Office of the Comptroller of the Currency \\
\hline OCIO & Office of the Chief Information Officer \\
\hline OCSP & Online Certificate Status Protocol \\
\hline ODBC & Open Database Connectivity \\
\hline OECD & Organisation for Economic Co-operation and Development \\
\hline OEM & original equipment manufacturer \\
\hline OFB & output feedback (mode) \\
\hline OFDM & orthogonal frequency-division multiplexing \\
\hline OGSA $^{\text {TM }}$ & Open Grid Services Architecture \\
\hline OHA & Open Handset Alliance \\
\hline OIG & Office of Inspector General \\
\hline OLE & object linking and embedding \\
\hline OMB & Office of Management and Budget \\
\hline ONS & Object Naming Service \\
\hline OOB & out-of-band \\
\hline OPC & OLE for Process Control \\
\hline OpenPGP & An Open Specification for Pretty Good Privacy \\
\hline OPM & U.S. Office of Personnel Management \\
\hline ORB & open relay blacklist \\
\hline OS & operating system \\
\hline OSHA & Occupational Safety and Health Administration \\
\hline OSI & Open Systems Interconnection \\
\hline OSPF & Open Shortest Path First \\
\hline OSS & open source software \\
\hline OSSTMM & Open Source Security Testing Methodology Manual \\
\hline OSVDB & Open Source Vulnerability Database \\
\hline OTP & one-time password \\
\hline OU & organizational unit \\
\hline OVAL & Open Vulnerability and Assessment Language \\
\hline OWASP & Open Web Application Security Project \\
\hline OWL-S & Web Ontology Language for Services \\
\hline \multicolumn{2}{|l|}{$\mathbf{P}$} \\
\hline $\mathbf{P 2 P}$ & peer-to-peer \\
\hline$P A C$ & Privilege Attribute Certificate \\
\hline$P A C$ & Protected Access Credential \\
\hline PAD & peer authorization database \\
\hline PAM & pluggable authentication module \\
\hline PAN & personal area network \\
\hline PAOS & Reverse HTTP Binding for SOAP \\
\hline$P A P$ & Password Authentication Protocol \\
\hline $\boldsymbol{P A P}$ & policy access point \\
\hline PAS & publicly available specification \\
\hline PBA & pre-boot authentication \\
\hline PBAC & policy-based access control \\
\hline PBCC & Packet Binary Convolutional Code \\
\hline
\end{tabular}




\begin{tabular}{|c|c|}
\hline PBE & pre-boot environment \\
\hline PBX & private branch exchange \\
\hline $\mathbf{P C}$ & personal computer \\
\hline PCI & Payment Card Industry \\
\hline PCI & personal identity verification card issuer \\
\hline PCI DSS & Payment Card Industry Data Security Standard \\
\hline PCMCIA & Personal Computer Memory Card International Association \\
\hline PCN & process control network \\
\hline PCP & IP Payload Compression Protocol \\
\hline PCS & process control system \\
\hline PCSF & Process Control System Forum \\
\hline PCSRF & Process Control Security Requirements Forum \\
\hline PDA & personal digital assistant \\
\hline PDD & Presidential Decision Directive \\
\hline PDF & Portable Document Format \\
\hline PDP & policy decision point \\
\hline PDS & protective distribution systems \\
\hline PEAP & Protected Extensible Authentication Protocol \\
\hline PED & portable electronic devices \\
\hline PEM & Privacy Enhanced Mail \\
\hline PEP & policy enforcement point \\
\hline PFS & perfect forward secrecy \\
\hline PGP & Pretty Good Privacy \\
\hline PHI & protected health information \\
\hline PHP & PHP: Hypertext Preprocessor \\
\hline PHY & Physical (layer) \\
\hline PIA & privacy impact assessment \\
\hline PICSTM $^{\text {TM }}$ & Platform for Internet Content Selection \\
\hline PII & personally identifiable information \\
\hline PIM & personal information management \\
\hline PIM-SM & Protocol Independent Multicast-Sparse Mode \\
\hline PIN & personal identification number \\
\hline PIP & policy information point \\
\hline PIR & Public Interest Registry \\
\hline PIV & personal identity verification \\
\hline PKCS & Public Key Cryptography Standard \\
\hline PKI & public key infrastructure \\
\hline PKM & privacy key management \\
\hline PKMv1 & Privacy Key Management Protocol version 1 \\
\hline PKMv2 & Privacy Key Management Protocol version 2 \\
\hline PL & public law \\
\hline PLC & programmable logic controller \\
\hline PMA & Policy Management Authority \\
\hline PMK & pairwise master key \\
\hline PMKSA & Pairwise Master Key Security Association \\
\hline PMP & point-to-multipoint \\
\hline PMTU & path maximum transmission unit \\
\hline $\mathbf{P N}$ & packet number \\
\hline PNG & Portable Network Graphics \\
\hline POA\&M & plan of action and milestones \\
\hline$P O C$ & point of contact \\
\hline
\end{tabular}




\section{POC \\ PoE \\ POP \\ POP3 \\ PP \\ PPP \\ PPTP \\ PPVPN \\ PRA \\ Pre-PAK \\ PRF \\ PRNG \\ PSK \\ PSTN \\ PTA \\ PTK \\ PTV \\ PUB \\ PUK \\ PVG}

Q

QoP

QoS

quality of protection

quality of service

$\mathbf{R}$

R\&D

R/W

$R A$

$R A$

$\boldsymbol{R A}$

$R A$

RAdAC

RADIUS

RAID

RAM

RAT

RBAC

RC2

RC4

RCE

RCFL

RCP

RDBMS

RDP

REL

REP

research and development

$\mathrm{read} / \mathrm{write}$

receiver address

Registration Authority

remote assistance

Router Advertisement

risk adaptive access control

Remote Authentication Dial In User Service

redundant array of independent disks

random access memory

remote administration tool

role-based access control

Rivest Cipher 2

Rivest Cipher 4

route cache entry

Regional Computer Forensics Laboratory

Remote Copy Protocol

relational database management system

Remote Desktop Protocol

rights expression language

Robots Exclusion Protocol 


\begin{tabular}{|c|c|}
\hline REST & Representational State Transfer \\
\hline RF & radio frequency \\
\hline RFC & request for comments \\
\hline RFD & route flap damping \\
\hline RFID & radio frequency identification \\
\hline RFP & request for proposal \\
\hline RIB & routing information base \\
\hline RIP & Routing Information Protocol \\
\hline RIPE NCC & Réseaux IP Européens Network Coordination Centre \\
\hline RIPng & Routing Information Protocol next generation \\
\hline RIR & regional internet registries \\
\hline RIS & Remote Installation Services \\
\hline RMA & reliability, maintainability, and availability \\
\hline RMON & Remote Monitoring \\
\hline RNG & random number generator \\
\hline ROE & rules of engagement \\
\hline ROM & read-only memory \\
\hline $\mathbf{R P}$ & responsible person (record) \\
\hline RPC & remote procedure call \\
\hline RPF & Reverse Path Forwarding \\
\hline RPO & recovery point objective \\
\hline $\mathbf{R R}$ & resource record \\
\hline RRSIG & resource record signature \\
\hline$R S$ & relay station \\
\hline$R S$ & Router Solicitation \\
\hline RSA & Rivest-Shamir-Adelman \\
\hline RSBAC & rule set-based access control \\
\hline RSN & Robust Security Network \\
\hline RSNA & Robust Security Network Association \\
\hline RSNIE & Robust Security Network Information Element \\
\hline RSO & reduced sign-on \\
\hline RSS & Really Simple Syndication \\
\hline RSSI & received signal strength indication \\
\hline RSVP & Resource ReSerVation Protocol \\
\hline RTF & Rich Text Format \\
\hline RTLS & real-time location system \\
\hline RTO & recovery time objective \\
\hline RTP & Real-Time Transport Protocol \\
\hline RTU & remote terminal unit or remote telemetry unit \\
\hline RuBAC & rule-based access control \\
\hline R-UIM & Removable User Identity Module \\
\hline \multicolumn{2}{|l|}{$\mathbf{S}$} \\
\hline S/MIME & Secure/Multipurpose Internet Mail Extensions \\
\hline$S A$ & security association \\
\hline$S A$ & source address \\
\hline SACL & system access control list \\
\hline SAD & security association database \\
\hline SAFER & Secure And Fast Encryption Routine \\
\hline
\end{tabular}




\begin{tabular}{|c|c|}
\hline SAID & security association identifier \\
\hline SAISO & senior agency information security officer \\
\hline$S A M$ & Security Account Manager \\
\hline$S A M$ & software asset management \\
\hline SAMATE & Software Assurance Metrics and Tool Evaluation \\
\hline SAMLTM & Security Assertion Markup Language ${ }^{\mathrm{TM}}$ \\
\hline SAN & storage area network \\
\hline S-BGP & Secure Border Gateway Protocol \\
\hline SC & subcommittee \\
\hline SCADA & supervisory control and data acquisition \\
\hline SCAP & Security Content Automation Protocol \\
\hline SCP & Secure Copy Protocol \\
\hline SCSI & Small Computer System Interface \\
\hline SCTP & Stream Control Transmission Protocol \\
\hline SD & Secure Digital \\
\hline SDIO & Secure Digital Input Output \\
\hline SDK & software development kit \\
\hline SDLC & System Development Life Cycle \\
\hline SDO & standards development organization \\
\hline$S D P$ & Session Description Protocol \\
\hline$S D P$ & Service Discovery Protocol \\
\hline SEI & Software Engineering Institute \\
\hline SEM & security event management \\
\hline SEND & Secure Neighbor Discovery \\
\hline SEP & secure entry point \\
\hline SFTP & Secure File Transfer Protocol \\
\hline SHA & Secure Hash Algorithm \\
\hline SHA-1 & Secure Hash Algorithm 1 \\
\hline shim6 & Site Multihoming by IPv6 Intermediation \\
\hline SHS & Secure Hash Standard \\
\hline SIA & Security Industry Association \\
\hline SID & security identifier \\
\hline SIEM & security information and event management \\
\hline SIG & special interest group \\
\hline SIIT & Stateless IP/ICMP Translation Algorithm \\
\hline SIM & security information management \\
\hline SIM & subscriber identity module \\
\hline SIP & Session Initiation Protocol \\
\hline SIS & safety instrumented system \\
\hline SKEME & Secure Key Exchange Mechanism \\
\hline SLA & service level agreement \\
\hline SMB & Server Message Block \\
\hline SME & subject matter expert \\
\hline S/MIME & Secure/Multipurpose Internet Mail Extensions \\
\hline SMS & Short Message Service \\
\hline SMS & Systems Management Server \\
\hline SMT & scar, mark and tattoo \\
\hline SMTP & Simple Mail Transfer Protocol \\
\hline SNL & Sandia National Laboratories \\
\hline SNMP & Simple Network Management Protocol \\
\hline SNTP & Simple Network Time Protocol \\
\hline
\end{tabular}




\begin{tabular}{|c|c|}
\hline SOA & service-oriented architecture \\
\hline SOA & start of authority (resource record) \\
\hline soBGP & Secure Origin Border Gateway Protocol \\
\hline SoD & separation of duties \\
\hline SOHO & small office/home office \\
\hline SOP & standard operating procedure \\
\hline SOR & system of records \\
\hline SORN & system of records notice \\
\hline SOX & Sarbanes-Oxley Act of 2002 \\
\hline$S P$ & service pack \\
\hline$S P$ & special publication \\
\hline SPD & security policy database \\
\hline SPI & security parameters index \\
\hline SPL & Structured Product Labeling \\
\hline SPMLTM & Service Provisioning Markup Language ${ }^{\mathrm{TM}}$ \\
\hline SPP-ICS & System Protection Profile for Industrial Control Systems \\
\hline SQL & Structured Query Language \\
\hline SR & service release \\
\hline SRES & signed response \\
\hline SRTP & Secure Real-Time Transport Protocol \\
\hline SS & subscriber station \\
\hline SSDP & Simple Service Discovery Protocol \\
\hline SSE-CMM & Systems Security Engineering-Capability Maturity Model \\
\hline SSH & Secure Shell \\
\hline SSI & Server Side Includes \\
\hline SSID & service set identifier \\
\hline SSL & Secure Sockets Layer \\
\hline SSLF & Specialized Security-Limited Functionality \\
\hline SSN & social security number \\
\hline SSO & single sign-on \\
\hline SSoD & static separation of duty \\
\hline SSP & secure simple pairing \\
\hline SSPI & Security Support Provider Interface \\
\hline ST & security target \\
\hline STA & station \\
\hline STIG & security technical implementation guide \\
\hline STS & security token service \\
\hline SUID & Set-User-ID \\
\hline SWSA & Semantic Web Services Initiative Architecture \\
\hline SZ & security zone \\
\hline \multicolumn{2}{|l|}{$\mathbf{T}$} \\
\hline TA & test assertion \\
\hline $\boldsymbol{T A}$ & transmitter address \\
\hline TACACS & Terminal Access Controller Access Control System \\
\hline TAG & technical advisory group \\
\hline TB & terabyte \\
\hline TC & technical committee \\
\hline
\end{tabular}




$\begin{array}{ll}\text { TC68 } & \text { ISO/IEC Technical Committee } 68 \\ \text { TCP } & \text { Transmission Control Protocol } \\ \text { TCP/IP } & \text { Transmission Control Protocol/Internet Protocol } \\ \text { TDEA } & \text { Triple Data Encryption Algorithm } \\ \text { TDM } & \text { time division multiplexing } \\ \text { TDMA } & \text { time division multiple access } \\ \text { TEK } & \text { traffic encryption key } \\ \text { TERENA } & \text { Trans-European Research and Education Networking Association } \\ \text { TFT } & \text { thin film transistor } \\ \text { TFTP } & \text { Trivial File Transfer Protocol } \\ \text { TGS } & \text { ticket-granting service } \\ \text { TIA } & \text { Telecommunications Industry Association } \\ \text { TID } & \text { tag identifier } \\ \text { TK } & \text { temporal key } \\ \text { TKIP } & \text { Temporal Key Integrity Protocol } \\ \text { TLD } & \text { top-level domain } \\ \text { TLS } & \text { Transport Layer Security } \\ \text { TMOVS } & \text { Modes of Operation Validation System for the Triple DES Algorithm } \\ \text { TOE } & \text { target of evaluation } \\ \text { TOS } & \text { trusted operating system } \\ \text { ToS } & \text { Type of Service } \\ \text { TPC } & \text { transmission power control } \\ \text { TPM } & \text { trusted platform module } \\ \text { TR } & \text { technical report } \\ \text { TRT } & \text { transport relay translator } \\ \text { TS } & \text { technical specification } \\ \text { TSA } & \text { time stamping authority } \\ \text { TSC } & \text { TKIP sequence counter } \\ \text { TSIG } & \text { Secret Key Transaction Authentication for DNS } \\ \text { TSIG } & \text { Transaction Signature } \\ \text { TSN } & \text { transitional security network } \\ \text { TSP } & \text { Time-Stamp Protocol } \\ \text { TT\&E } & \text { test, training, and exercise } \\ \text { TTF } & \text { tag talks first } \\ \text { TTL } & \text { time to live } \\ \text { TTLS } & \text { Tunneled Transport Layer Security } \\ \text { TTP } & \\ \text { TXT } & \end{array}$

U.S.

U.S.C.

UAC

UART

UBR

UCC

UCE

UDDITM

UDF
United States

United States Code

User Account Control

universal asynchronous receiver/transmitter

Universal Description, Discovery and Integration (UDDI) Business Registry

Uniform Code Council, Inc.

unsolicited commercial email

Uniform Description, Discovery, and Integration ${ }^{\mathrm{TM}}$

Universal Disk Format 


\begin{tabular}{|c|c|}
\hline UDP & User Datagram Protocol \\
\hline UFS & UNIX File System \\
\hline UHF & ultra high frequency \\
\hline UI & user interface \\
\hline UK & United Kingdom \\
\hline $\mathbf{U L}$ & Underwriters’ Laboratories ${ }^{\circledR}$ \\
\hline ULA & unique local address \\
\hline ULP & upper layer protocol \\
\hline UML@ & Unified Modeling Language ${ }^{\mathrm{TM}}$ \\
\hline UMPC & ultra-mobile personal computer \\
\hline UMTS & Universal Mobile Telecommunications System \\
\hline UNII & Unlicensed National Information Infrastructure \\
\hline UPC & Universal Product Code \\
\hline UPnP & Universal Plug and Play \\
\hline UPS & uninterruptible power supply \\
\hline URI & Uniform Resource Identifier \\
\hline URL & Uniform Resource Locator \\
\hline USB & Universal Serial Bus \\
\hline US-CERT & United States Computer Emergency Readiness Team \\
\hline USIM & UMTS Subscriber Identity Module or Universal Subscriber Identity Module \\
\hline UTM & unified threat management \\
\hline UUID & Universally Unique Identifier \\
\hline UWB & ultrawideband \\
\hline \multicolumn{2}{|l|}{$\mathbf{V}$} \\
\hline VB & Visual Basic \\
\hline VB.NET & Visual Basic .NET \\
\hline VBA & Visual Basic for Applications \\
\hline VBScript & Visual Basic Script \\
\hline VFD & variable frequency drive \\
\hline VHD & virtual hard drive \\
\hline VHF & very high frequency \\
\hline VLAN & virtual local area network \\
\hline VM & virtual machine \\
\hline VMS & vulnerability management system \\
\hline VoIP & Voice over Internet Protocol \\
\hline VOIPSA & Voice over IP Security Alliance \\
\hline VPN & virtual private network \\
\hline VPNC & Virtual Private Network Consortium \\
\hline VRRP & Virtual Router Redundancy Protocol \\
\hline \multicolumn{2}{|l|}{$\mathbf{W}$} \\
\hline W3C $₫$ & World Wide Web Consortium \\
\hline WAN & wide area network \\
\hline$W A P$ & wireless access point \\
\hline$W A P$ & Wireless Application Protocol \\
\hline WaSP & Web Standards Project \\
\hline
\end{tabular}




$\begin{array}{ll}\text { WAVE } & \text { Wireless Access for Vehicular Environment } \\ \text { WAYF } & \text { Where Are You From } \\ \text { WCCP } & \text { Web Cache Coordination Protocol } \\ \text { W-CDMA } & \text { Wideband Code Division Multiple Access } \\ \text { WDS } & \text { wireless distribution system } \\ \text { WebDAV } & \text { Web Distributed Authoring and Versioning } \\ \text { WEP } & \text { Wired Equivalent Privacy } \\ \text { WfMC } & \text { Workflow Management Coalition } \\ \text { WfMS } & \text { workflow management system } \\ \text { WG } & \text { working group } \\ \text { WIDPS } & \text { wireless intrusion detection and prevention system } \\ \text { WiMAX } & \text { Worldwide Interoperability for Microwave Access } \\ \text { WLAN } & \text { wireless local area network } \\ \text { WMAN } & \text { wireless metropolitan area network } \\ \text { WMM } & \text { Wi-Fi Multimedia } \\ \text { WORM } & \text { write once, read many } \\ \text { WPA } & \text { Wi-Fi Protected Access } \\ \text { WPA2 } & \text { Wi-Fi Protected Access }{ }^{\circledR} 2 \\ \text { WPAN } & \text { wireless personal area network } \\ \text { WS } & \text { Web services } \\ \text { WSDL } & \text { Web Services Description Language } \\ \text { WSH } & \text { Windows Script Host } \\ \text { WS-I } & \text { Web services interoperability } \\ \text { WS-I } & \text { Web Services Interoperability Organization } \\ \text { WSS4J } & \text { Web Services Security for Java } \\ \text { WS-Security } & \text { Web Services Security } \\ \text { WSUS } & \text { Windows Server Update Services } \\ \text { WVE } & \text { Wireless Vulnerabilities and Exploits } \\ \text { WWAN } & \text { wireless wide area network } \\ \text { WWW } & \text { World Wide Web } \\ & \end{array}$

$\mathrm{XYZ}$

XACL

XACMLTM

$\mathrm{XCBC}$

$\mathrm{XCCDF}$

XHTML

XKMS

XML

XOR

XrML

XSD

XSL

XSLT

XSS

ZSK
XML Access Control Language

eXtensible Access Control Markup Language ${ }^{\mathrm{TM}}$

XOR Cipher Block Chaining

eXtensible Configuration Checklist Description Format

Extensible Hypertext Markup Language

XML Key Management Specification

Extensible Markup Language

exclusive OR

eXtensible Rights Markup Language

XML Schema Definition

Extensible Stylesheet Language

Extensible Stylesheet Language Transformation

cross-site scripting

zone signing key 


\section{Appendix A-References}

Sources used in the development of the list of system and network security acronyms and abbreviations in this document include the following:

National Institute of Standards and Technology Publications, NIST Computer Security Division Resource Center Web site, http://csrc.nist.gov/

Internet Engineering Task Force (IETF), http://www.ietf.org/

Microsoft Hardware Developer Central, Glossary of Acronyms for PC and Server Technologies, http://www.microsoft.com/whdc/resources/support/glossary.mspx

Organization for the Advancement of Structured Information Standards (OASIS), http://www.oasis-open.org/home/index.php 


\section{Appendix B-Former Acronyms}

Over time, as organizations, technologies, or other entities change, some acronyms associated with them may lose their definitions and thus no longer be considered acronyms. This appendix presents selected former acronyms related to system and network security. As additional acronyms in this publication lose their definitions, readers are encouraged to send notification of these, along with references to authoritative sources of information, to securityacronyms@ nist.gov for possible inclusion in future releases of this report.

IEEE

Originally defined as "Institute of Electrical and Electronics Engineers, Inc." Definition dropped by the organization (http://www.ieee.org/web/aboutus/home/index.html).

SOAP

Originally defined as "Simple Object Access Protocol." Definition dropped as of April 2007 (http://www.w3.org/TR/soap12-part1/\#intro). 